ORIGINAL ARTICLE

\title{
Unexplained extra visits to general practitioners before the diagnosis of first urinary tract infection: a case-control study
}

\author{
J H van der Voort, A G Edwards, R Roberts, R G Newcombe, K Verrier Jones
}

See end of article for authors' affiliations

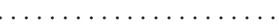

Correspondence to: DrJ van der Voort Assistant Professor and Staff Paediatric

Nephrologist, Children's Hospital, Room 3G49, McMaster Medical Centre, 1200 Main Street West, Hamilton, Ontario L8S 4J9, Canada;

jhvandervoort@hotmail.com

Accepted 29 June 2002

\begin{abstract}
Aims: To determine: (1) whether children diagnosed with a urinary tract infection (UTI) visited their general practitioner (GP) more frequently before the diagnosis of UTI was established compared to children never diagnosed with a UTI; and (2) whether those children with evidence of renal scarring at their first diagnosed UTI visited their GPs more frequently before diagnosis compared to children who did not have evidence of renal scarring when their first UTI was investigated.

Methods: Case-control study of 77 children with a UTI identified from a hospital radiology database (37 with and 40 without renal scarring), and 77 age, sex, and general practice matched controls. Main outcome measures were entries in general practice clinical records for types of illness, antibiotic prescriptions, and urine samples requested prior to the diagnosis of first UTI (cases) or equivalent time periods for controls.

Results: Cases had a mean 2.94 additional visits or $21 \%$ more visits $(95 \% \mathrm{Cl} 1 \%$ to $41 \%)$ in the period (mean 2.4 years) prior to the visit at which their first UTI was diagnosed, including a mean 2.5 additional visits or $23 \%$ more visits for infectious illness $(95 \% \mathrm{Cl} 1 \%$ to $45 \%)$. The cases had $114 \%$ (95\% $\mathrm{Cl} 41 \%$ to $184 \%$ ) more visits for symptoms relating to the genitourinary tract, though the actual number of these visits was small. They were febrile at $49 \%$ more visits $(95 \% \mathrm{Cl} 1 \%$ to $99 \%)$ and received significantly more courses of antibiotics than controls $(5.2 \mathrm{v} 4.1)$. They had more urine samples requested (37 v 3). Both the cases with and without renal scarring had similar excess GP visits.

Conclusion: Compared to controls, children diagnosed with a first UTI had more visits at which symptoms of infection were recorded and more antibiotics prescribed prior to the visit at which the first UTI was diagnosed. These excess visits may have included undiagnosed UTIs. Both those with and without renal scarring had a similar degree of excess visits; additional aetiological factors must have played a role in scar formation.
\end{abstract}

U rinary tract infections (UTIs) are most common in the first years of life, but reported incidences vary by country and setting. Studies in general practice in the UK found the incidence in children under 2 years of age ranged from $0 \%$ to $4 \% .{ }^{12}$ A population based study from Sweden found a cumulative diagnostic rate of $1.6 \%$ in all children less than 2 years. ${ }^{3}$ Some of this variation in incidence may be explained by variation in the definition of a UTI and sampling methods. However, lack of awareness of the epidemiology and presentation of UTIs in infants and toddlers and lack of facilities, equipment, and skill in urine collection in primary care probably also contribute. ${ }^{4}$ The extent of under diagnosis of UTI in early childhood remains unknown.

UTIs cause acute morbidity and may result in permanent renal scarring, leading to hypertension and reduced renal function. ${ }^{5}$ Delay in diagnosis, young age, vesicoureteric reflux (VUR), and recurrent infections have all been implicated in the aetiology of renal scarring. ${ }^{67}$ There are theoretical and practical reasons why children should benefit from earlier diagnosis and treatment of UTI. UTIs in young children are often recurrent: $33 \%$ of children with their first UTI under 1 year of age had a recurrence within two years. ${ }^{8}$ With earlier diagnosis, babies and toddlers with symptoms of UTI are more likely to be managed in a way that reduces prolonged suffering, uncertainty about the diagnosis, parental anxiety, recurrent visits to the general practitioner (GP), and referral to hospital with undiagnosed illness. Parents and doctors can diagnose subsequent UTIs earlier, and antibiotic prophylaxis can potentially be started. ${ }^{9}$ Recurrent UTIs, particularly if VUR is present, can cause reflux nephropathy (RN) and occasion- ally renal failure. This may be prevented by early recognition and treatment. ${ }^{10}{ }^{11} \mathrm{RN}$ is irreversible, but about $5-10 \%$ of children with UTI have scarring already present when first diagnosed with a UTI. ${ }^{12}{ }^{13}$ The proportion of children with renal failure caused by $\mathrm{RN}$ varies from country to country: in Sweden, a country with a high awareness of UTI, RN is now a rare cause of end stage renal disease, ${ }^{14}$ whereas in the UK, RN is still a common cause of renal failure. ${ }^{15}$

Strategies to reduce acute morbidity of UTIs and the incidence of RN should focus on early diagnosis of the first UTI. This study explored the hypotheses that: (1) children diagnosed with a UTI had an excess of GP consultations for illness episodes compatible with possible UTIs in early childhood compared to controls; and (2) those children with renal scarring present at diagnosis of the first UTI had more evidence of undiagnosed illness and fevers, compared with children diagnosed with a UTI but no renal scarring present when first diagnosed. We studied the period before the first UTI was diagnosed using a case-control study design.

\section{PATIENTS AND METHODS}

Patient selection

The University Hospital of Wales and Cardiff Royal Infirmary hospital radiology databases were searched for consecutive

Abbreviations: GP, general practitioner; RN, reflux nephropathy; UTI, urinary tract infection; VUR, vesicoureteric reflux 
Table 1 Mean number of GP visits for cases prior to the visit at which a first UTI was diagnosed and for matched controls for an equivalent time period

\begin{tabular}{|c|c|c|c|c|}
\hline Type of visit & Mean cases & Mean controls & Adjusted difference (with 95\% CI) & $\begin{array}{l}\text { Difference as \% of average visits } \\
\text { (with } 95 \% \mathrm{CI} \text { ) }\end{array}$ \\
\hline All visits & 15.5 & 12.9 & $+2.9(+0.1 \text { to }+5.8)^{*}$ & $+21 \%(+1 \text { to }+41 \%)^{*}$ \\
\hline Trauma & 0.26 & 0.62 & $-0.38(-0.8$ to +0.1$)$ & $-86 \%(-191$ to $+18 \%)$ \\
\hline All symptoms of infection & 12 & 9.7 & $+2.5(+0.13 \text { to }+4.92)^{*}$ & $+23 \%(+1 \text { to }+45 \%)^{*}$ \\
\hline URTI symptoms & 6.0 & 5.1 & $+1.0(-0.49$ to +2.56$)$ & $+18 \%$ (-9 to +46\%) \\
\hline Gastrointestinal symptoms & 1.22 & 1.14 & $+0.1(-0.39$ to +0.65$)$ & $+11 \%(-33$ to $+55 \%)$ \\
\hline GU symptoms & 0.49 & 0.14 & $+0.4(+0.13 \text { to }+0.58)^{* *}$ & $+114 \%(+41$ to $+184 \%) * *$ \\
\hline Febrile illness & 1.05 & 0.65 & $+0.4(+0.01 \text { to }+0.84)^{*}$ & $+49 \%(+1 \text { to }+99 \%)^{*}$ \\
\hline
\end{tabular}

children investigated by DMSA scan after a microbiologically proven and treated first UTI from 1992 until 1997. Urine samples were obtained by clean catch or urine collection bags. No samples were obtained by suprapubic aspiration or urethral catheterisation. Urine samples were cultured with the filter paper method, using CLED medium, incubating at $35-37^{\circ} \mathrm{C}$ for 24 hours. A proven UTI was defined as a growth of a single organism of more than $100000 / \mathrm{ml}$. Renal scarring was defined as: one or more focal, discrete scar(s), unilateral or bilateral global scarring, or abnormal differential function of less than $43 \%$ on one side. Children were excluded if they had congenital abnormalities of the renal tract (except VUR). We asked their GPs to identify the next child on the practice register of the same sex and within six months of the age of the case (as a matched control). The GP notes of both cases and controls were photocopied and anonymised (by RR). Any reference to the diagnosis and treatment of the first UTI in the notes was removed. The study was approved by the Bro Taf Local Research Ethics Committee. Directed by the GP, consent to examine the children's general practice records was obtained from parents.

\section{Data extraction}

Using a diagnostic tree for general practice related illnesses, two blinded researchers ( JvdV and AE) independently coded all GP consultations (excluding health surveillance and immunisation visits) between the day of registration with the practice and the day before the diagnosis of the first UTI for cases, or for an equal time period for their matched controls. Differences in coding of illness episodes were discussed between the two researchers and resolved by consensus. The total number of consultations was recorded as well as the reason for the consultation: consultations were subdivided into those for symptoms suggesting infectious illness and those where infectious illness was unlikely. Consultations were further subdivided into symptoms of infection of different anatomical sites, for example, the respiratory tract, gastrointestinal tract, or genitourinary tract. Temperature status of children was categorised as febrile, afebrile, or unrecorded. Prescribed courses of antibiotics and number of urine samples requested for culture were counted. Socioeconomic status was imputed using the Townsend score, based on postal code.

\section{Statistical analysis}

The total number of consultations per child was compared between case and control groups by paired analysis of covariance, adjusting for the Townsend score as a covariate. Similar analyses were performed for each category of visit. Percentage differences shown in table 1 relate to the average of the mean for the case and control groups. Using data from a previous pilot study, we calculated that 80 cases and 80 controls were required to detect a $25 \%$ difference in visits at a $5 \%$ significance level with an $80 \%$ power.

\section{RESULTS}

Searches identified 77 cases, of which 40 had a normal DMSA scan and 37 had evidence of renal scarring on DMSA scan. Seventy seven matched controls were identified. The study population included 96 girls (48 cases and 48 controls). All patients were registered with a GP before 3 months of age, apart from four who registered between 4 and 6 months of age. The mean duration of follow up was 28.6 months (range 1-112 months). The mean Townsend scores for cases and controls were 1.10 and 1.4 respectively. This meant that cases were less socially deprived than controls. Eighty entries (3.4\%, 28 for cases, 52 for controls) of the total number of entries (2202) were uninterpretable.

Table 1 shows the mean number of GP consultations for cases and controls. Cases visited their GP significantly more often than controls $(\mathrm{p}<0.05)$ in the study period and most of these additional visits were for symptoms of infectious illness $(p<0.05)$, for example, gastrointestinal symptoms, upper respiratory tract symptoms, urinary tract symptoms $(p<0.01)$, and visits for which a fever was recorded $(p<0.05)$. In contrast, cases visited less often for minor trauma. They were prescribed $27 \%$ more courses of antibiotics: 5.2 courses per case and 4.1 courses per control $(\mathrm{p}<0.05)$. In total 40 urine samples were requested from 1381 consultations for symptoms suggestive of infection (3\%). No urine sample was requested on a child less than 1 year of age. The cases had more urine samples requested: 0.48 samples per case ( 37 in total for cases) were requested prior to the diagnosis of the first UTI, compared to 0.04 samples per control (three samples in total for controls) for the matched time

Table 2 Characteristics of cases (patients diagnosed with UTI) with and without renal scarring on DMSA scan

\begin{tabular}{lll}
\hline & Scarred $(n=37)$ & Non-scarred $(n=40)$ \\
\hline Age at presentation of 1st UTI & 29.4 months & 27.9 months \\
Patients (\%) admitted to hospital with 1st UTI & $20(54)$ & $23(58)$ \\
Patients (\%) presenting with fever & $26(70)$ & $26(65)$ \\
Patients (\%) presenting with vomiting & $13(35)$ & $13(33)$ \\
VUR grade 3 or higher & $9 / 40$ renal units & $1 / 30$ renal units \\
\hline
\end{tabular}


period. Of these requested urine collections, only 20 results $(50 \%)$ could be traced, two of which showed a definite UTI but were not recognised as such or acted on (one by a GP, one by a hospital). Both these children had renal scarring present when diagnosed with a UTI at a later point.

The 37 UTI cases with renal scarring on DMSA scan had a mean of 13.7 consultations before UTI diagnosis and the 40 cases without renal scarring had a mean of 17.2 consultations. This difference was not statistically significant, but the sample size was small. There was no difference between those children with and without renal scarring for admission rates, age at diagnosis of first UTI, presentation, and duration of symptoms (presence of fever or vomiting). However those children who had renal scarring were more likely to have grade 3 or higher VUR, according to the classification of the international study of kidney diseases in children (table 2). ${ }^{16}$

\section{DISCUSSION}

We wanted to explore whether UTIs might have occurred but were not diagnosed in primary care in the period preceding the first diagnosed UTI. The additional visits to primary care for infectious illness among the children with subsequent UTI lends support to this hypothesis. Our second aim was to examine whether renal scarring was associated with an excess of visits compared to children diagnosed with a UTI but without evidence of renal scarring. This study showed that possible undiagnosed UTIs precede the UTI that brings a child with renal scarring to the attention of medical staff. However the mean number of additional visit was no larger than in a child with no renal scarring. We therefore did not find support for the hypothesis that undiagnosed UTIs alone cause renal scarring.

The main strength of this study lies in the use of an entire population of sequential cases from two regional hospital databases and the rigorous examination of the GP records. Researchers from both child health and general practice disciplines independently undertook the blinded analysis of the anonymised records. Although the study was dependent on the quality of the mainly handwritten GP records, few data entries were uninterpretable. Data were extracted only for broad categories of illness episode. Evidence for mean additional visits was found for all infectious illness categories, but not for visits obviously not related to infections. The broad categorisation of illness episodes therefore does not appear to have limited adequate testing of the hypotheses. The case-control study design allowed us to efficiently explore these hypotheses.

Weaknesses of this study include us not taking into consideration family size, birth order, or day care attendance as possible confounding factors. These factors are known to be associated with an increased incidence of infectious illness. It is possible that out of hours consultations or consultations to other emergency services were not always recorded in the general practice notes and might therefore have been missed.

UTI is most common in the first two years of life and can have long term sequelae. The diagnostic rate in Sweden is higher than in the UK, especially in those paediatric centres with a high level of awareness. ${ }^{3}$ UTIs are often not recognised in febrile young children in primary care because of lack of awareness by health professionals and parents, and practical difficulties with urine collection in primary care. ${ }^{4}$ Jadresic et al showed that the more GPs send urine samples from children, the higher the diagnostic rate in that practice. ${ }^{17}$ The presence of another potential source of fever, such as upper respiratory tract infection or otitis media, is not reliable in excluding UTI in children under 2 years of age. ${ }^{18}$

We explored whether UTIs may have been classified under other specific disease categories by GPs. All categories for symptoms of infection showed increased visits for cases compared to controls, especially febrile symptoms and symptoms of the urinary tract. Minor trauma visits were less frequent in these cases, in keeping with less social deprivation on Townsend scores, which suggest validity of our data. This also suggests that cases did not simply have a lower threshold for consulting overall. The GPs asked for a urine culture more frequently in the study group, but the number of urine cultures requested was smaller than guidelines suggest appropriate. A result could be traced for only half of the requests, suggesting that in some cases no sample was collected or that samples did not reach the laboratory. Earlier diagnosis of UTI could have been made and treatment given to two children with positive urine samples that were not acted on and who were later found to have renal scarring. Cases were prescribed more courses of antibiotics than controls. This may have treated some undiagnosed UTIs but hindered recognition of the true diagnosis. This study therefore supports our hypothesis that UTIs may not have been diagnosed in early childhood in our study population. Alternatively, a factor may be present that leads to increased susceptibility to infections in various anatomical systems, including the urinary tract.

The additional visits to the GP were no different between cases with and without renal scars. However, the presence of more severe reflux in this subgroup may have accounted for the development of renal scars in some of these children. ${ }^{19}$ Although bacterial virulence and host resistance factors may account for the development of scarring in children without VUR, ${ }^{20}{ }^{21}$ we did not find evidence that the diagnosed first UTIs in the scarring group were more pyelonephritic in nature: age distribution, symptoms, and admission rates were equal among those cases with and without renal scars. The study therefore supports the hypothesis that UTIs may have been missed in early childhood in children with renal scarring, but that other unidentified factors play an additional role in scar formation. In addition, this study lends justification for a large prospective cohort study.

\section{ACKNOWLEDGEMENTS}

This study was supported by a grant from Kidney Research Unit for Wales Foundation. We thank Mrs Jo Baker and Mrs Pat Griffiths for secretarial assistance and Prof. Chris Butler for excellent editorial assistance. We thank all participating GPs and practice managers for their help with the study.

\section{Authors' affiliations}

J H van der Voort, KRUF Children's Kidney Centre, University Hospital of Wales, Cardiff CF14 4XW, UK

A G Edwards, Department of General Practice, University of Wales

College of Medicine, Heath Park, Cardiff CF14 4XN, UK

R Roberts, Department of Child Health, University of Wales College of

Medicine

R G Newcombe, K Verrier Jones, Department of Epidemiology,

Statistics and Public Health, University of Wales College of Medicine

\section{REFERENCE}

1 Dickinson JA. Incidence and outcome of symptomatic urinary tract infection in children. BM 1979:1:1330-2.

2 Brooks D, Houston IB. Symptomatic urinary infection in childhood: presentation during a four-year study in general practice and significance and outcome at seven years. J R Coll Gen Pract 1977;27:678-83.

3 Jakobsson B, Esbjorner E, Hansson S. Minimum incidence and diagnostic rate of first urinary tract infection. Pediatrics 1999; 104:222-6

4 van der Voort JH, Edwards A, Roberts R, et al. The struggle to diagnose urinary tract infection in children under two in primary care. Family Practice 1997; 14:44-8

5 Jacobson $\mathrm{SH}$, Eklof O, Eriksson CG, et al. Development of hypertension and uraemia after pyelonephritis in childhood: 27 year follow up. BM 1989:299:703-6.

6 Berg UB, Johansson SB. Age as a main determinant of renal functional damage in urinary tract infection. Arch Dis Child 1983;58:963-9.

7 Smellie JM, Poulton A, Prescod NP. Retrospective study of children with renal scarring associated with reflux and urinary infection. BM 1994;308: $1193-6$ 
8 Nuutinen $M$, Uhari $M$. Recurrence and follow-up after urinary tract infection under the age of 1 year. Pediatr Nephrol 2001;16:69-72.

9 Smellie JM, Katz G, Gruneberg RN. Controlled trial of prophylactic treatment in childhood urinary tract infection. Lancet 1978;2:175-8.

10 Verber IG, Meller ST. Serial $99 \mathrm{mTc}$ dimercaptosuccinic acid (DMSA) scans after urinary infections presenting before the age of 5 years. Arch Dis Child 1989;64:1533-7.

11 Ransley PG, Risdon RA. Reflux nephropathy: effects of antimicrobial therapy on the evolution of the early pyelonephritic scar. Kidney Int 1981:20:733-42.

12 Deshpande PV, Verrier Jones K. An audit of RCP guidelines on DMSA scanning after urinary tract infection. Arch Dis Child 2001;84:324-7.

13 Sreenarasimhaiah S, Hellerstein S. Urinary tract infections per se do not cause end-stage kidney disease. Pediatr Nephrol 1998;12:210-13.

14 Esbjorner E, Berg U, Hansson S. Epidemiology of chronic renal failure in children: a report from Sweden 1986-1994. Pediatr Nephrol $1997 ; 11: 438-42$.
15 Imam A, Roberts R, Verrier Jones K. Chronic renal failure in children in Wales: a prospective epidemiological study 1994-1997 [abstract]. Pediatr Nephrol 1998;12:C182.

16 International Reflux Study Committee. International system of radiographic grading of vesico-ureteric reflux. Pediatr Radiol 1985;15:105-9.

17 Jadresic L, Cartwright K, Cowie N, et al. Investigations of urinary tract infection in childhood. BM 1993;307:761-4.

18 Shaw KN, Gorelick M, McGowen KL, et al. Prevalence of urinary tract infection in febrile young children in the emergency department. Pediatrics 1998;102:e16.

19 Goldman M, Bistritzer T, Horne T, et al. The etiology of renal scars in infants with pyelonephritis and vesicoureteral reflux. Pediatr Nephrol 2000;14:385-8.

20 Lomberg $\mathbf{H}$, Jodal $\mathrm{U}$, Leffler $\mathrm{H}$, et al. Blood group non-secretors have an increased inflammatory response to urinary tract infection. Scand J Infect Dis 1992:24:77-83.

21 Svanborg-Eden C, De Mann P, Jodal U, et al. Host parasite interaction in urinary tract infection. Pediatr Nephrol 1987;1:623-31.

\section{IMAGES IN PAEDIATRICS}

\section{Toe deformity from prolonged pulse oximetry}

W have seen toe deformities in three of our babies who needed prolonged pulse oximetry. The deformities started to manifest beyond the age of 3 months. They consisted of elevation of one or more of the second to fourth toes (see figs 1 and 2 ) and persisted in the three children at the ages of 12,18 , and 30 months respectively, though not while weight bearing in the latter.

There are well described complications of pulse oximetry, including burns ${ }^{1}$ and finger injury. ${ }^{2}$ We are, however, not aware of any previous documentation of toe deformity as a possible complication.

This deformity, although possibly transient, causes concern to the parents and health care staff. Some of the possible explanations for the deformities could include how firmly the probes are being applied or the frequency with which they are re-sited. The use of splints to support the toes during oximetry may help to prevent the deformities.

We have started placing the probes more proximally on the feet and are re-siting them at three hourly intervals instead of 4-6 hourly. This is a preventable consequence and we should therefore be cautious and vigilant in our use of pulse oximetry.

D Lindo, D Browne

Neonatal Unit, Kingston Hospital, Galsworthy Road, Kingston upon Thames, Surrey KT2 7QB UK; dlindo@kingstonhospital.nhs.uk

J Lindo

Department of Public Health Sciences, St George's Hospital Medical School, London, UK

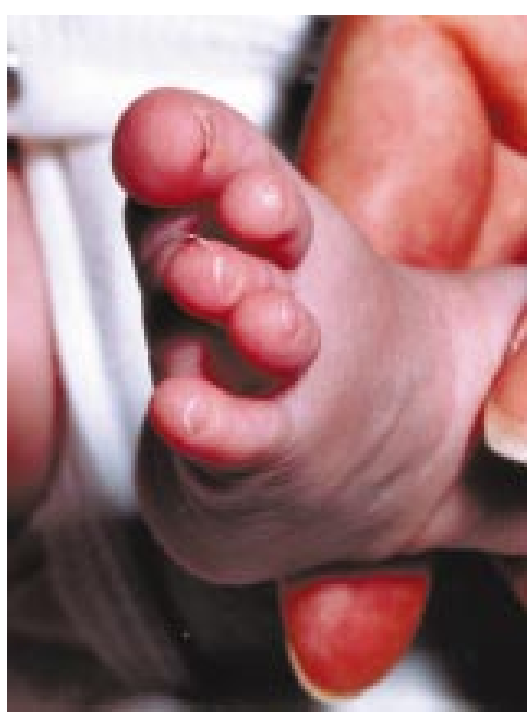

Figure 1

\section{References}

1 Murphy KG, Secunda JA, Rockoff MA. Severe burns from pulse oximeter. Anesthesiology 1990;73:350-2.

2 Wille J, Braams R, Haren W, et al. Pulse oximeter-induced digital injury: frequency rate and possible causitive factors. Crit Care Med 2000;28:3555-7.

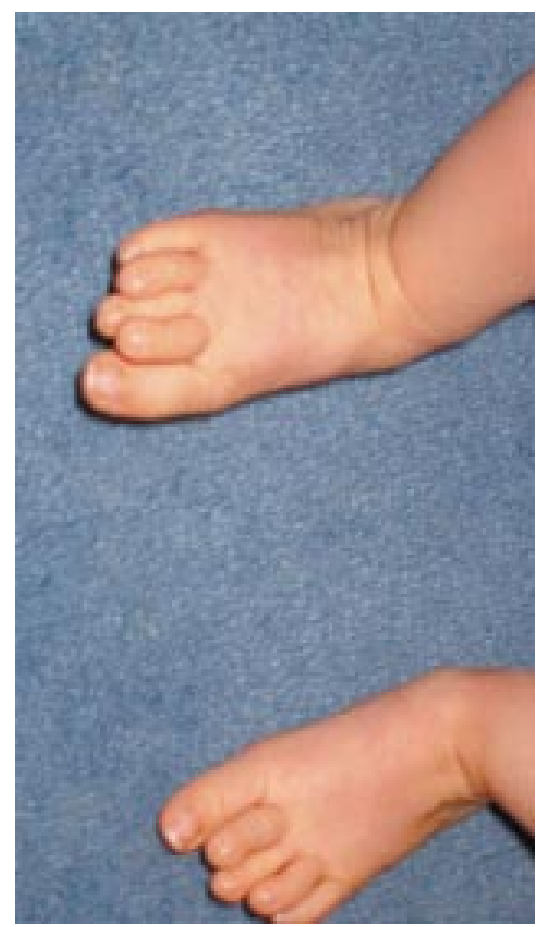

Figure 2 